\title{
ON AN ELEMENTARY ANALOGUE OF THE RIEMANN-MANGOLDT FORMULA
}

\author{
AUREL WINTNER
}

Ramanujan's unsuccessful approach to the Prime Number Theorem, published only recently, is based on the power series

$$
\sum_{n=1}^{\infty} \Lambda(n) x^{n} \equiv \sum_{n} \log p \sum_{k=1}^{\infty} x^{p^{k}} \quad \text { and } \quad \sum_{n=1}^{\infty} p^{n} x^{p^{n}}, \quad 0<x<1,
$$

where $p$ denotes a prime and $p$ is 2 in the last series. In his discussion of Ramanujan's failure in case of the latter series, a series impracticable as $x \rightarrow 1$, Hardy gives for the function represented by the series another expansion, one exhibiting the critical "wobbles," as follows: ${ }^{1}$

$$
\sum_{n=1}^{\infty} p^{n} \exp \left(-p^{n} s\right)=\{\} / \log p, \quad p=2,
$$

where \{\} is the expression

$$
\begin{aligned}
\{\}=\frac{1}{s} & -\frac{1}{s} \cdot \sum_{k=-\infty}^{\infty} \Gamma\left(\frac{1+2 \pi k i}{\log p}\right) s^{-2 \pi k i / \log p} \\
& -\log p \sum_{n=0}^{\infty} \frac{(-1)^{n} p^{n+1}}{p^{n+1}-1} \frac{s^{n}}{n !}
\end{aligned}
$$

it being understood that $\sum_{k=-\infty}^{\infty \prime}=\sum_{k=-\infty}^{-1}+\sum_{k=1}^{\infty}$ and $-\log x=s>0$.

It will be seen later on that Hardy's result (2) contains two errors. However, the purpose of this note is not calculation of the corrections necessary, which are of a trivial nature, but the presentation of a short approach which seems to be of methodical and historical interest.

First, (2) is of the same type as the "explicit formula" of RiemannMangoldt ${ }^{2}$ (the two sums representing the contributions of the "nontrivial" and "trivial" zeros, respectively). Correspondingly, Hardy's

Received by the editors January 12, 1942.

${ }^{1}$ G. H. Hardy, Ramanujan, Cambridge, 1940, chap. II, formulae (2.9.1) and (2.11.2).

2 Cf., for example, A. E. Ingham, The Distribution of Primes, Cambridge Tracts, no. 30 (1932), chap. IV.

Relevant for the comparison is only the "Abelian" form (instead of the deeper "Cesàro" form) of the Riemann-Mangoldt formula; cf. G. H. Hardy and J. E. Littlewood, Acta Mathematica, vol. 41 (1918), pp. 119-196. 
proof, omitted loc. cit., ${ }^{1}$ is based on a contour integration. ${ }^{3}$ However, since the "non-trivial" zeros occurring in the part $\sum$ ' of (2) are equidistant, the "wobbly" terms now become purely periodic, indicating the possibility of a trivial approach. ${ }^{4}$

It turns out that such an approach has been found already by Dedekind. What makes it of particular interest is the fact that Dedekind's purpose was precisely the explicit illustration of the illegitimacy of the argument to occur decades later in Ramanujan's attempted proof of the Prime Number Theorem. Inasmuch as Dedekind's result, published in a certainly conspicuous work, ${ }^{5}$ has never ${ }^{6}$ been quoted, it is possible that his indications were found to be hard to follow. All of this seems to warrant a detailed and straightforward deduction of the explicit formula.

To this end, put

$$
f(s)=\sum_{n=-\infty}^{\infty} c^{n} \exp \left(-c^{n} s\right)
$$

where $c$ is any constant satisfying $c>1$. Then (3) obviously represents a regular function ${ }^{7}$ for $s>0$. It is also seen that (3) remains unchanged if $n$ is replaced by $n+1$ in the exponentials; so that ${ }^{8} s f(s)$ remains unaltered if $s$ is replaced by $c s$. It follows that, if $\log c>0$ and $s>0$,

$$
f(s) \log c=s^{-1} \sum_{k=-\infty}^{\infty} \Gamma(1+C k i) s^{-C k i}, \quad \text { where } C=2 \pi / \log c
$$

(it being understood that $s^{\alpha i}$ is meant to be defined in terms of the principal branch of $\log s)$.

In fact, since $s f(s)=c s f(c s)$, the function $e^{u} f\left(e^{u}\right)$ of the real variable $u=\log s$ has the period $\log c$. Since this function, being regular, is continuous and of bounded variation, the Fourier expansion

${ }^{3}$ G. H. Hardy, Lectures on the Mathematical Work of Ramanujan, The Institute for Advanced Study, 1936, p. 14.

${ }^{4} \mathrm{Cf}$. Ingham's comments on $\sum$ ' $\sin 2 \pi n x / n$, loc. cit., p. 81 .

${ }^{5}$ Supplement IX of Dirichlet's Vorlesungen ueber Zahlentheorie (pp. 385-386 of the 3rd edition (1879)).

The non-differentiable function of Weierstrass depends on a limiting case of Dedekind's series. In this connection, cf. G. H. Hardy and J. E. Littlewood, Proceedings of the National Academy of Sciences, vol. 2 (1916), pp. 583-586.

${ }^{6} \mathrm{Cf}$. Hardy's paper of 1907 , quoted loc. cit., Footnote 1, p. 47, $\$ 2.11$.

7 It will always be assumed that $s>0$, the transition from the half-line $s>0$ to the half-plane $R(s)>0$ being trivial.

8 This remark of Dedekind corresponds to the more elaborate argument (loc. cit., Footnote 1, p. 39) which Hardy's paper of 1907 attributes to Wedderburn (cf. loc. cit., Footnote 6). 
where

$$
e^{u} f\left(e^{u}\right)=\sum_{k=-\infty}^{\infty} \alpha_{k} e^{-C k i u}
$$

$$
\alpha_{k}=\frac{1}{\log c} \int_{0}^{\log c} e^{C k i u} e^{u} f\left(e^{u}\right) d u, \quad C=\frac{2 \pi}{\log c}
$$

is valid for every $u=\log s$. Hence, in order to prove (4), it is sufficient to verify that the last integral is $\Gamma(1+C k i)$. But the uniformity of the convergence of the series (3) assures that this integral is

$$
\sum_{n=-\infty}^{\infty} c^{n} \int_{0}^{\log c} e^{C k i u} e^{u} \exp \left(-c^{n} e^{u}\right) d u
$$

or, since $\int_{0}^{a} F(u) d u \equiv \int_{n a}^{(n+1) a} F(u-n a) d u$ and $C=2 \pi / \log c$,

$$
\sum_{n=-\infty}^{\infty} c^{n} \int_{n \log c}^{(n+1) \log c} e^{C k i u} e^{u-n \log c} \exp \left(-c^{n} e^{u-n \log c}\right) d u,
$$

that is,

$$
\sum_{n=-\infty}^{\infty} c^{n} \int_{n \log c}^{(n+1) \log c} e^{C k i u} e^{u} c^{-n} \exp \left(-c^{n} e^{u} c^{-n}\right) d u
$$

or simply

$$
\sum_{n=-\infty}^{\infty} \int_{n \log c}^{(n+1) \log c} e^{(1+C k i) u} \exp \left(-e^{u}\right) d u \equiv \int_{-\infty}^{\infty} e^{(1+C k i) \mathfrak{u}} \exp \left(-e^{u}\right) d u .
$$

Since the last integral is reduced by $u=\log s$ to the integral defining $\Gamma(1+z)$ for $z=C k i$, the proof of the Dedekind expansion (4) is complete.

While the (real) series (3) converges only" for $s>0$, its "non-principal part," that is, the series

$$
g(s)=\sum_{m-1}^{\infty} c^{-m} \exp \left(-c^{-m} s\right),
$$

converges for every $s$, since $c>1$. Furthermore, the function (5) has the Taylor series

$$
g(s)=\sum_{n=0}^{\infty} \frac{(-1)^{n}}{c^{n+1}-1} \frac{s^{n}}{n !}
$$

${ }^{9}$ Incidentally, the function (3) of the complex variable $s$, where $R(s)>0$, has the line $R(s)=0$ as natural boundary. In fact, (3) and (5) show that $f(s)-g(s)$ is a lacunary Dirichlet series for every $c>1$. On the other hand, (5) or (6) implies that $g(s)$ is an entire function. 
for every $s$. In fact, substitution of the geometric progression

$$
\left(c^{n+1}-1\right)^{-1}=\sum_{m=1}^{\infty} c^{-(n+1) m,} \quad c>1,
$$

transforms (6) into an iterated series which, being absolutely convergent, can be rearranged into

$$
\sum_{m=1}^{\infty} \sum_{n=0}^{\infty}(-1)^{n} c^{-(n+1) m} s^{n} / n !
$$

But this may be written in the form

$$
\sum_{m=1}^{\infty} c^{-m} \sum_{n=0}^{\infty}\left(-c^{-m} s\right)^{n} / n !
$$

which is identical with (5).

According to (3) and (5),

$$
\sum_{n=0}^{\infty} c^{n} \exp \left(-c^{n} s\right)=f(s)-g(s)
$$

If (4) and (6) are substituted into (7), the product of the Dirichlet series (7) into $s$ appears as decomposed into a Fourier series in $\log s$ and an entire power series in $s$.

If the power series of $\exp (-s)$ is added to (5) and (6), then (7) appears in the form

$$
\sum_{n=1}^{\infty} c^{n} \exp \left(-c^{n} s\right)=f(s)-\sum_{n=0}^{\infty} \frac{(-1)^{n} c^{n+1}}{c^{n+1}-1} \frac{s^{n}}{n !} .
$$

Hence, (4) implies Hardy's explicit formula (2) for (1), if $-\sum^{\prime}$ is corrected to $+\sum$ ' and

$$
\Gamma\left(\frac{1+2 \pi k i}{\log p}\right) \text { to } \Gamma\left(1+\frac{2 \pi k i}{\log p}\right) .
$$

The Johns Hopkins University 